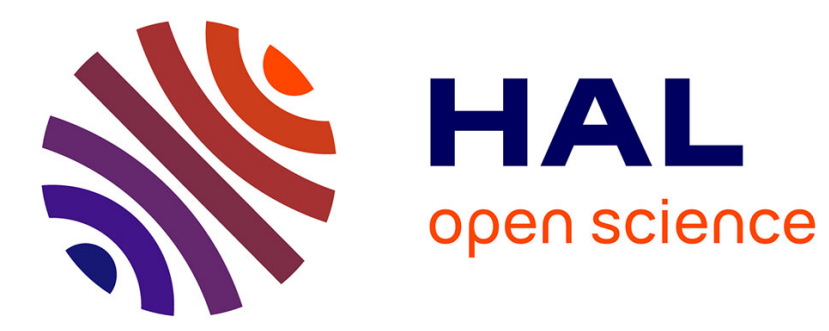

\title{
Cinquième partie. - Propriétés magnétiques des amorphes ioniques
}

\author{
G. Ferey
}

\section{To cite this version:}

G. Ferey. Cinquième partie. - Propriétés magnétiques des amorphes ioniques. Revue de Physique Appliquée, 1980, 15 (6), pp.1043-1048. 10.1051/rphysap:019800015060104300 . jpa-00244821

\section{HAL Id: jpa-00244821 https://hal.science/jpa-00244821}

Submitted on 1 Jan 1980

HAL is a multi-disciplinary open access archive for the deposit and dissemination of scientific research documents, whether they are published or not. The documents may come from teaching and research institutions in France or abroad, or from public or private research centers.
L'archive ouverte pluridisciplinaire HAL, est destinée au dépôt et à la diffusion de documents scientifiques de niveau recherche, publiés ou non, émanant des établissements d'enseignement et de recherche français ou étrangers, des laboratoires publics ou privés. 


\title{
Cinquième partie. - Propriétés magnétiques des amorphes ioniques
}

\author{
G. Ferey \\ Laboratoire des Fluorures et Oxyfluorures Ioniques (E.R.A. 609), \\ Faculté des Sciences de l'Université du Maine, route de Laval, 72017 Le Mans Cedex
}

1. Introduction. - Les composés ioniques, cristallisés ou amorphes, nécessitent la combinaison d'un ou plusieurs métaux électropositifs avec un élément très électronégatif. Ce dernier point limite le plus souvent le domaine d'étude aux fluorures et dans une moindre mesure aux oxydes pour lesquels l'effet de covalence est déjà marqué.

L'étude de systèmes binaires et ternaires de fluorures et d'oxydes a montré depuis longtemps l'existence de domaines vitreux [1-17]. Ils mettent en jeu des matrices diamagnétiques : $\mathrm{BeF}_{2}, \mathrm{AlF}_{3}, \mathrm{ZrF}_{4}, \mathrm{ThF}_{4}, \mathrm{SiO}_{2}$, $\mathrm{GeO}_{2}, \mathrm{Al}_{2} \mathrm{O}_{3}, \mathrm{~B}_{2} \mathrm{O}_{3}, \mathrm{P}_{4} \mathrm{O}_{10}$, etc., susceptibles de dissoudre des teneurs variables en éléments magnétiques, en particulier $\mathrm{Fe}_{2} \mathrm{O}_{3}$. Plus récemment des composés magnétiques amorphes de composition définie ont pu être isolés, et permettent ainsi d'étudier le magnétisme des amorphes sur des modèles simples : citons par exemple $\mathrm{FeF}_{2}$ [18-20], $\mathrm{FeF}_{3}$ [21, 22], $\mathrm{Fe}_{2} \mathrm{O}_{3}, \mathrm{Y}_{3} \mathrm{Fe}_{5} \mathrm{O}_{12}, \mathrm{CoFe}_{2} \mathrm{O}_{4}$ [23-25]. Après quelques remarques préliminaires sur les conséquences de l'état ionique pour les amorphes, nous nous attacherons ensuite à caractériser les divers types de magnétisme observés sur les composés définis amorphes.

2. Conséquences de l'état ionique [27]. - Dans l'état solide, chaque type de liaison présente des caractéristiques qui déterminent la configuration des proches voisins. Dans l'état ionique la nécessité d'une neutralité électrique locale implique en particulier un environnement exclusivement anionique pour les cations. Cette simple remarque est susceptible d'apporter des renseignements, tant au niveau des modèles structuraux envisageables que sur les interactions magnétiques dans ce type de matériaux :

2.1 Du Point De VUe structural. - Lorsque l'amorphe contient des ions de transition, le champ de ligands agit sur le cation qui joue alors le rôle de sonde locale; le spectre d'absorption du composé [26], caractéristique de la coordinence du cation de transition, fournira alors une information supplémentaire lors de l'élaboration d'un modèle structural pour le corps considéré.

2.2 Au NIVEAU MAGNÉTIQUE. - L'environnement anionique des cations paramagnétiques implique l'abandon des concepts d'échange direct ou du type
RKKY, qui supposaient un échange isotrope, uniquement fonction de la distance entre atomes. Il faut le remplacer par le modèle de superéchange qui dépend toujours de la distance entre ions magnétiques, mais aussi de l'angle de liaison cation-anion-cation [28-29]. Dans l'état amorphe, cet angle sera soumis à quelques variations qui resteront de faible amplitude pour une stœchiométrie et une coordinence cationique déterminées. Comme l'a décrit J. M. D. Coey [27], cette inéquivalence des sites conduit :

- à des distributions étroites des valeurs des moments magnétiques, puisque les moments ne sont pas très réduits par rapport aux valeurs de l'ion libre par effet de covalence avec les ligands :

- à une distribution des interactions d'échange à cause de la dépendence de $J$ vis-à-vis de la distance et de l'angle de liaison dans le superéchange. Le couplage entre paires de spins peut être exclusivement positif (ferromagnétisme). S'il est négatif ou qu'il présente les deux types de signe, diverses voies d'échange pourront alors conduire à différents alignements relatifs d'une paire; cet effet, connu sous le nom de frustration [31-33] est également présent pour certains composés cristallisés [34]. Il a été très discuté à propos du problème des verres de spin [35-38] en utilisant le modèle d'Edwards Anderson d'une distribution gaussienne, symétrique d'interactions d'échange autour de $J=0$. Il aboutit à un abaissement de la température d'ordre magnétique et peut conduire à des structures magnétiques non colinéaires dans les composés amorphes. La détermination de la température de gel, qui correspond au blocage des spins, dépend de la technique de mesure utilisée.

\section{Les types de magnétisme dans les amorphes} ioniques et leurs méthodes d'étude. - 3.1 LES COMPOSÉS DÉFINIS AMORPHES. - Leur mise en évidence est récente et ne permet pas encore une comparaison suffisamment complète avec les systèmes métalliques correspondants. Dans ces derniers, J. M. D. Coey établit une classification [27] qui lui permet de distinguer le magnétisme des alliages suivant le nombre de leurs sous-réseaux magnétiques, chaque sous-réseau étant défini comme un groupe d'atomes ayant des interactions magnétiques similaires. Il est amené à distinguer quatre cas théoriquement possibles dans les structures magnétiques à un sous-réseau (Fig. 1) : 


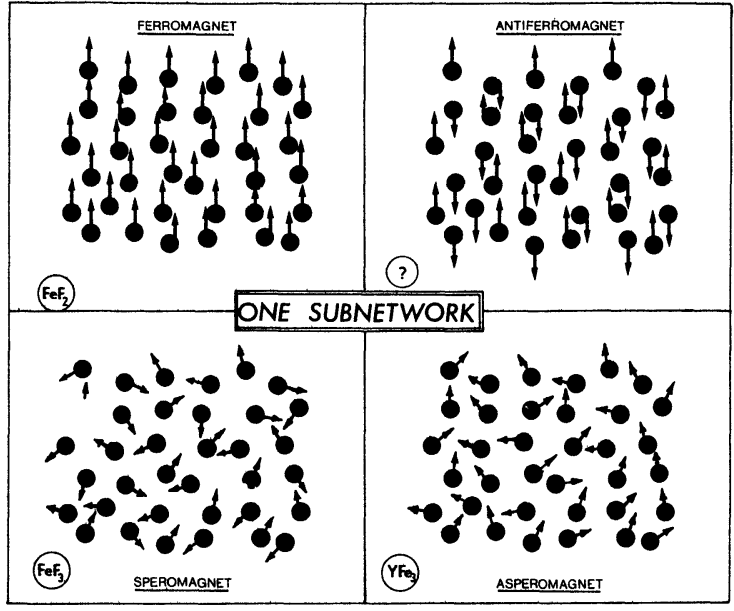

Fig. 1. - Les quatre structures magnétiques possibles pour un amorphe à un sous-réseau (d'après [27]).

[Possible one-subnetwork amorphous magnetic structures [27].]

- le ferromagnétisme

- l'antiferromagnétisme, en remarquant toutefois avec Simpson [39] qu'il est impossible de créer une réelle subdivision en deux sous-réseaux dans un solide non cristallin, l'antiferromagnétisme ne pourra donc exister au-delà de quelques distances interatomiques à cause des perturbations créées par le désordre topologique,

- le spéromagnétisme [40], qu'il définit comme une structure dans laquelle les spins sont gelés dans des directions aléatoires, de manière isotrope. La probabilité $P(\psi)$ pour qu'un spin fasse un angle $\psi$ avec une direction donnée est proportionnelle à $\sin \psi$,

- l'aspéromagnétisme [41] qui diffère du précédent par une distribution anisotrope des moments, avec quelques directions privilégiées. Aucun amorphe ionique ne montre jusqu'à présent ce dernier type de comportement, observé particulièrement sur $\mathrm{YFe}_{3}$ [42].

Deux amorphes ioniques rentrent dans cette classification : $\mathrm{FeF}_{2}$ [18-20] ferromagnétique et $\mathrm{FeF}_{3}$ spéromagnétique [21, 22]. Leur étude magnétique a été réalisée à partir de mesures d'aimantation effectuées sur magnétomètre à échantillon vibrant, de mesures de susceptibilité (méthode de Faraday) ainsi que des mesures de susceptibilité en champ très faible alternatif $(\sim 10 \mathrm{Oe})$.

a) $\mathrm{FeF}_{2}$. - Seul exemple de ferromagnétique isolant non cristallin, il constitue l'exemple typique de l'influence de la structure sur le magnétisme. La température de Curie donnée par Litterst [20] est de $21 \mathrm{~K}$ (Fig. 2), alors qu'à l'état cristallisé, $\mathrm{FeF}_{2}$ de structure rutile - est antiferromagnétique $\left(T_{\mathrm{N}}=80 \mathrm{~K}\right)$.

Entre 21 et $15 \mathrm{~K}$, les courbes $M=f(H / T)$ se superposent, révélant ainsi un comportement superparamagnétique (Fig. 3). Le moment voisin de $4 \mu_{\mathrm{B}}$ par ion fer $2+$ indique de plus un superparamagné-

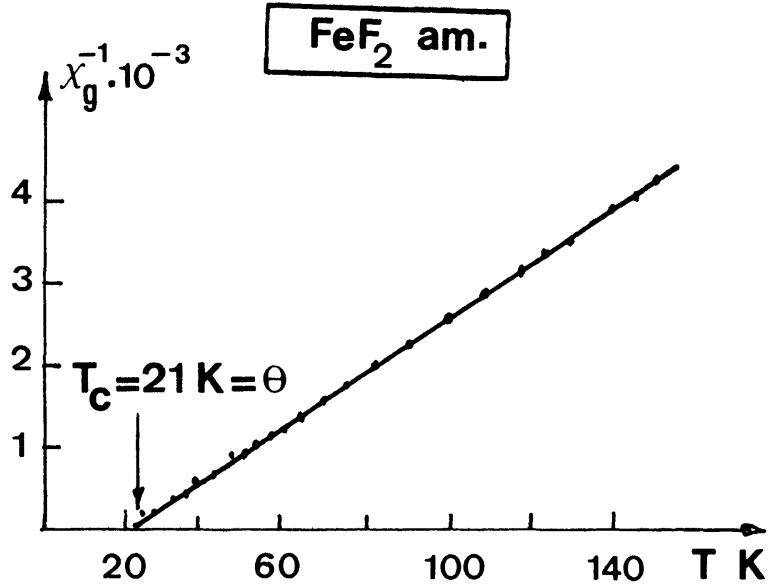

Fig. 2. - Variation thermique de la susceptibilité inverse de $\mathrm{FeF}_{2}$ amorphe (d'après [19]).

[Thermal variation of inverse susceptibility of amorphous $\mathrm{FeF}_{2}[19]$.]

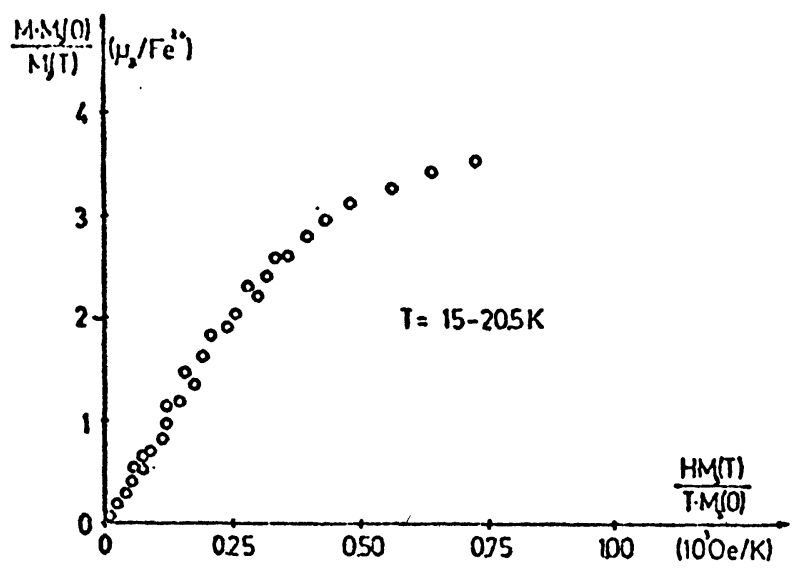

Fig. 3. - Courbes $M=f(H / T)$ entre 15 et $20,5 \mathrm{~K}$ pour $\mathrm{FeF}_{2}$ amorphe (d'après [20]).

$[M=f(H / T)$ curves between 15 and $20.5 \mathrm{~K}$ of amorphous $\left.\mathrm{FeF}_{2}[20].\right]$

tisme de configuration ferromagnétique, le volume des particules étant voisin de $1200 \AA^{3}$.

En dessous de $T_{\mathrm{c}}$, les courbes d'aimantation présentent (Fig. 4) de l'hystérésis et de la rémanence. Cette dernière, de l'ordre de $1,5 \mu_{\mathrm{B}}$ à $4,2 \mathrm{~K}$, décroît très lentement avec le temps.

b) $\mathrm{FeF}_{3}$. - Les propriétés magnétiques des deux variétés cristallisée et amorphe sont une nouvelle fois très différentes (Fig. 5); $\mathrm{FeF}_{3}$ amorphe ne présente d'ordre magnétique qu'à $T_{\mathrm{g}}=29 \mathrm{~K}$ au lieu de $T_{\mathrm{N}}=364 \mathrm{~K}$ pour $\mathrm{FeF}_{3}$ cristallisé. L'ensemble des mesures magnétiques effectuées sur $\mathrm{FeF}_{3}$ amorphe montre qu'il constitue l'illustration la plus stricte du spéromagnétisme [44]. Cela se manifeste expérimentalement par les faits suivants :

a) Une variation linéaire de l'aimantation avec le champ, quelle que soit la température (Fig. 6). 


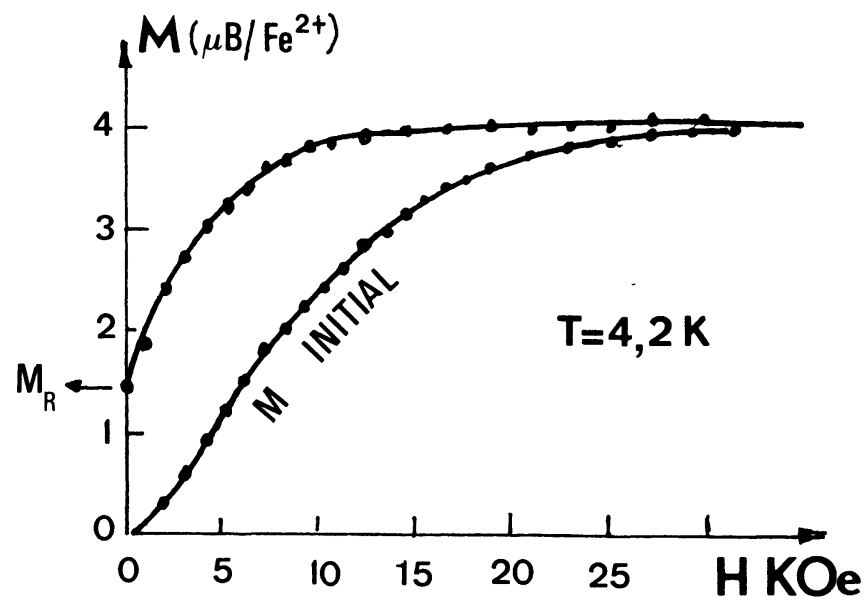

Fig. 4. - Aimantation de $\mathrm{FeF}_{2}$ non cristallin à 4,2 $\mathrm{K}$ (d'après [20]).

[Non crystalline $\mathrm{FeF}_{2}$ magnetization at $4.2 \mathrm{~K}$ [20].]

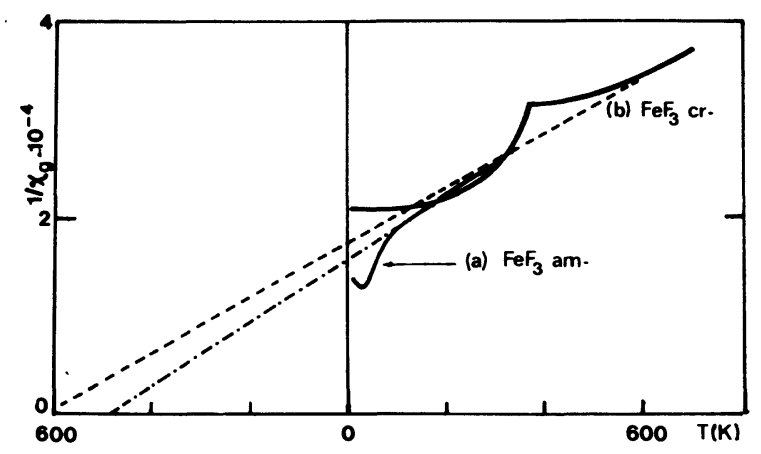

Fig. 5. - Variation thermique de la susceptibilité inverse des variétés amorphe (a) et cristallisée (b) de $\mathrm{FeF}_{3}$.

[Thermal variation of inverse susceptibility of amorphous $(a)$ and crystalline $(b) \mathrm{FeF}_{3}$.]

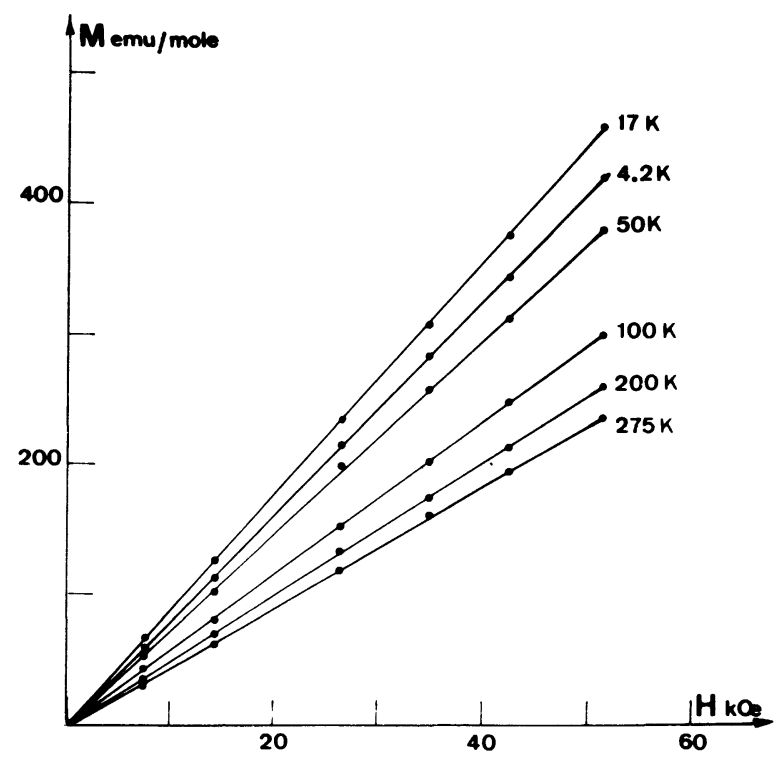

Fig. 6. - Courbes $\mathrm{M}(\mathrm{H})$ de $\mathrm{FeF}_{3}$ amorphe à diverses températures.

[M(H) curves of amorphous $\mathrm{FeF}_{3}$ at various temperatures.] $\beta)$ Un maximum très aplati de susceptibilité qui dépend peu du champ de mesure, même sous $10 \mathrm{Oe}$, contrairement aux verres de spin (Fig. 7).

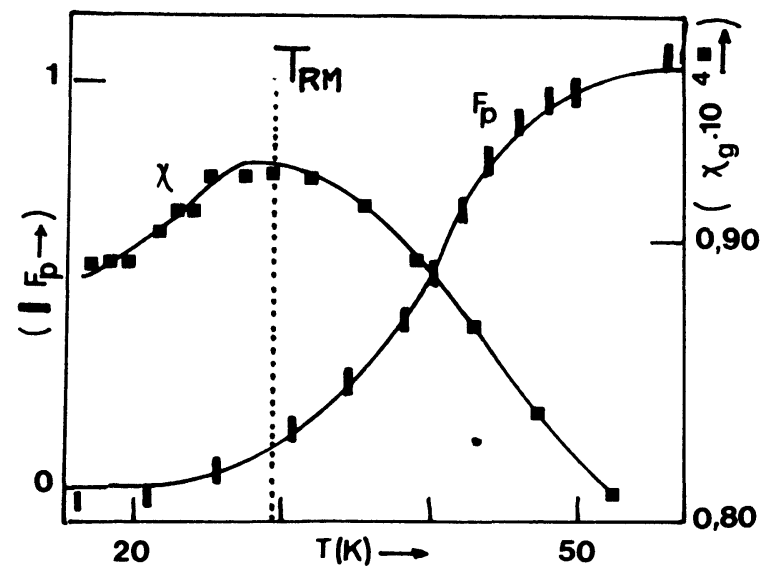

Fig. 7. - Variation thermique de la susceptibilité $\chi$ en champ faible $(10 \mathrm{Oe})$ et de la fraction paramagnétique $F_{\mathrm{p}} \mathrm{de}_{\mathrm{FeF}}$ amorphe (cf. Chapitre Varret-Henry)

[Thermal variation of low field $(\simeq 10 \mathrm{Oe})$ susceptibility and paramagnetic ratio of amorphous $\mathrm{FeF}_{3}$ (see chapter Varret-Henry).]

$\gamma)$ Une valeur absolue élevée de la température de Curie asymptotique $\left(\theta_{\mathrm{p}}=-486 \mathrm{~K}\right.$ pour $\left.\mathrm{FeF}_{3}\right)$, preuve d'interactions antiferromagnétiques fortes.

$\delta)$ Une température de gel magnétique faible devant $\theta_{\mathbf{p}}$ et une constante de Curie molaire très inférieure à la valeur théorique, ce qui indique une frustration intense dans l'arrangement des spins, et caractérise une distribution des intégrales d'échange sur chaque site (Fig. 6).

$\varepsilon$ ) L'observation d'aimantations rémanentes stables, fonction du champ magnétique appliqué lors du refroidissement de l'échantillon et de la mesure (Fig. 8) : en effet, au-dessous de $T_{\mathrm{g}}$, et quel que soit le champ appliqué pour la mesure, l'aimantation d'un échantillon refroidi sous champ nul est toujours inférieure à celle du même produit refroidi sous champ. Les deux aimantations deviennent égales audessus de $T_{\mathrm{g}}$.

Lorsqu'on coupe le champ, il subsiste une aimantation rémanente caractéristique $d u$ traitement antérieur :

1) L'aimantation thermorémanente (TRM) : obtenue en refroidissant à 4,2 $\mathrm{K}$ l'échantillon sous le champ de $17 \mathrm{kOe}$ dans notre exemple, puis en coupant ensuite le champ. L'aimantation résultante est la TRM. Sa température de disparition correspond à $T_{\mathbf{g}}$.

2) L'aimantation rémanente isotherme (IRM) : après refroidissement sous champ nul jusqu'à $4,2 \mathrm{~K}$, on applique le même champ - ici $17 \mathrm{kOe}$ - pour mesurer l'aimantation correspondante. Après la mesure, le champ est coupé. L'aimantation qui subsiste est l'IRM.

Ђ) Même à des champs très élevés, grande différence entre ces deux aimantations (Fig. 9). La satura- 


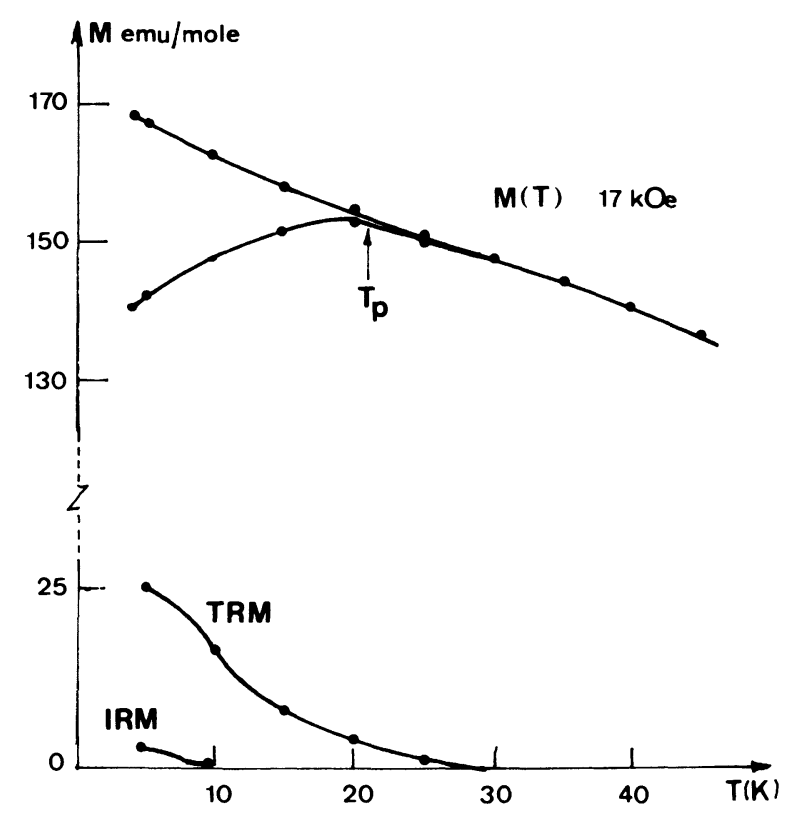

Fig. 8. - Evolution thermique de l'aimantation (M), de la thermorémanence (TRM) et de la rémanence isotherme (IRM) pour un champ de $17 \mathrm{kOe}$. L'échantillon est refroidi dans le champ de $60 \mathrm{~K}$ à la température de mesure pour la mesure de la TRM, alors qu'il est refroidi en champ nul pour l'IRM.

[Thermal evolution of magnetization (M), thermoremanent (TRM), isothermal remanent (IRM) magnetization of amorphous $\mathrm{FeF}_{3}$ under $17 \mathrm{kOe}$. The sample is cooled under the measuring field from $60 \mathrm{~K}$ to the measurement temperature for TRM, and cooled without field for IRM.]

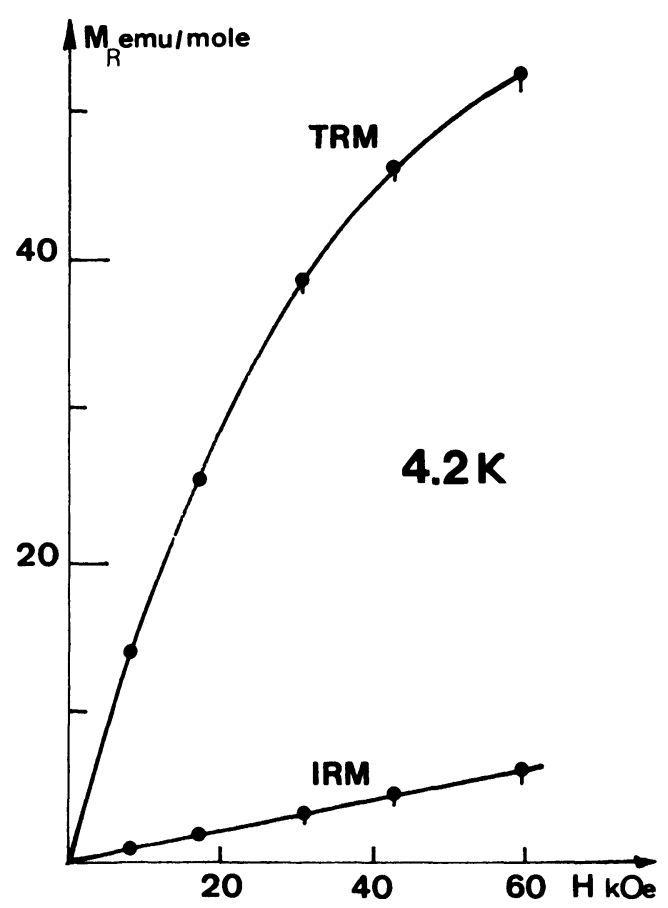

Fig. 9. - Variation avec le champ de la TRM et de l'IRM mesurées à $4,2 \mathrm{~K}$. La longueur des lignes verticales montre la variation de la rémanence après $5 \mathrm{~min}$.

[Variation of TRM and IRM vs. $\mathrm{H}$ at $4.2 \mathrm{~K}$. The vertical lines show the evolution of the remanence after $5 \mathrm{~min}$.] tion n'interviendrait qu'avec des champs de quelques centaines de kOe, contrairement aux verres de spin.

$\eta)$ L'apparition de cycles d'hystérésis en dessous de la température de gel (Fig. 10).

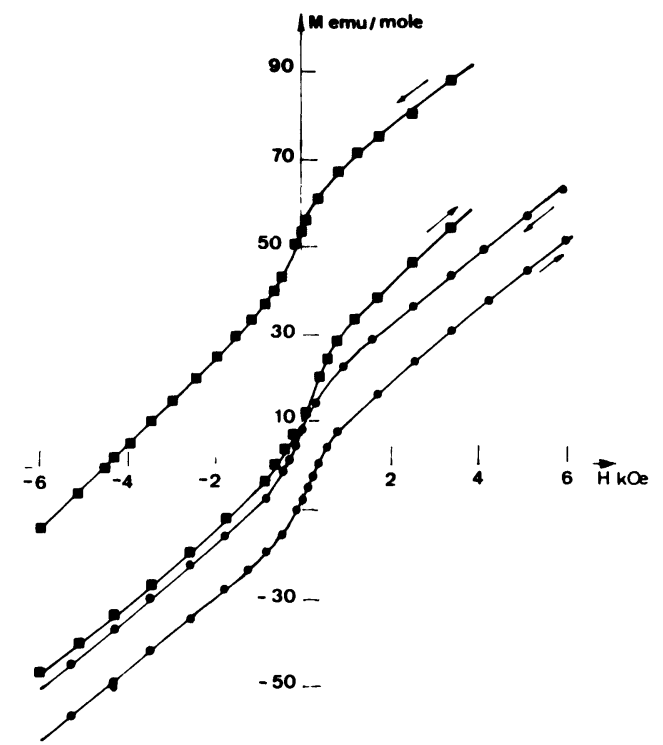

Fig. 10. - Portion centrale des cycles d'hystérésis sous $60 \mathrm{kOe}$ à $4,2 \mathrm{~K}$, montrant les effets de rémanence. Le cycle inférieur est obtenu en refroidissant en champ nul, alors que le cycle supérieur est déplacé à la suite du refroidissement sous $60 \mathrm{kOe}$.

[Central part of hysteresis loops under $60 \mathrm{kOe}$ at $4.2 \mathrm{~K}$, showing the remanence effects. The lower loop is obtained by cooling in zero field and the upper one after cooling under $60 \mathrm{kOe}$.]

Il n'existe pas actuellement d'équivalent ionique des alliages amorphes métalliques de terre rare et d'élément de transition à deux sous-réseaux qui mettent en évidence deux types de magnétisme (Fig. 11) :

- le ferrimagnétisme, observé dans $\mathrm{GdCo}_{3}$ [45] et $\mathrm{GdFe}_{2}$ [46-48],

- le spérimagnétisme justifié à partir de $\mathrm{DyCo}_{3}$ et $\mathrm{NdCo}_{3}$ [49-50].

3. 2 LES VERRES. - Ils sont obtenus à partir de mélanges binaires ou ternaires et se caractérisent par des compositions variables. Cette caractéristique pose immédiatement le problème de l'homogénéité des échantillons, surtout lorsque le verre contient des éléments magnétiques : ceux-ci se répartissent-ils uniformément dans le verre ou constituent-ils des amas magnétiques? Cette ambiguïté gêne considérablement l'interprétation des résultats magnétiques.

Ceux-ci sont par ailleurs très influencés par la concentration en ions magnétiques et donnent lieu à deux types de comportement :

- verre de spin [35-38], [52]

- mictomagnétisme [51].

Historiquement, ces deux vocables étaient utilisés pour désigner des composés cristallisés, principalement métalliques. Bien qu'il n'existe pas à l'heure actuelle d'exemple décisif de verre de spin ionique, nous rappellerons brièvement les propriétés les plus 


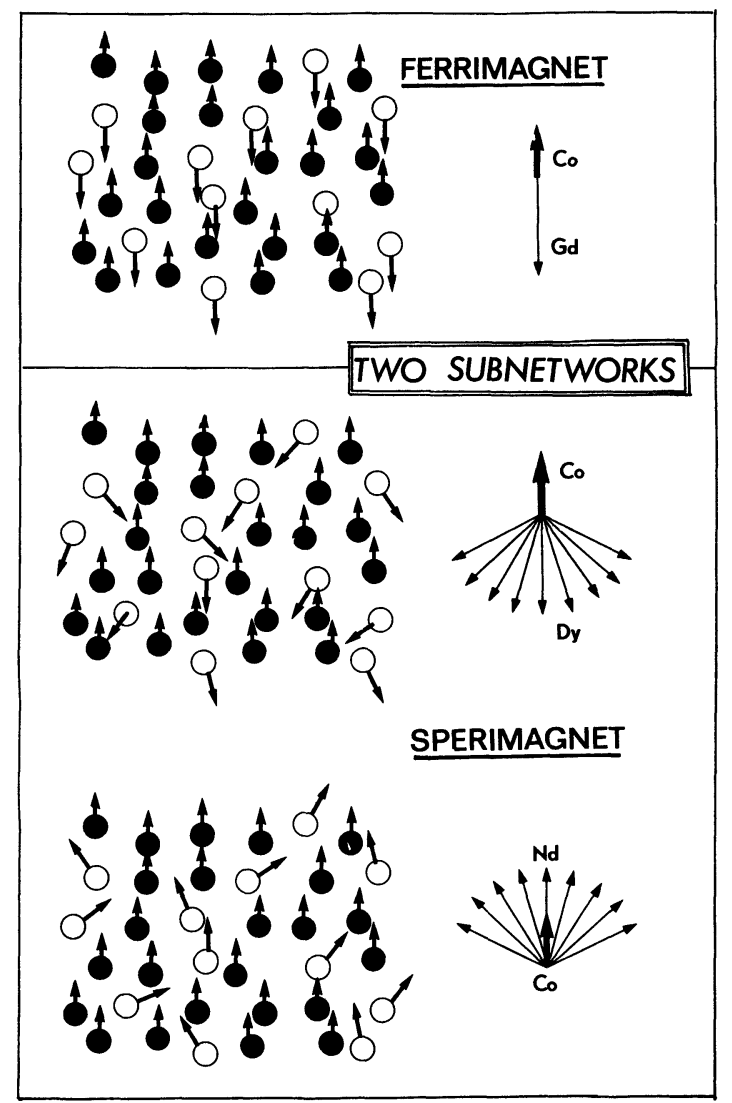

Fig. 11. - Les deux structures magnétiques possibles pour un amorphe à deux sous-réseaux (d'après J. M. D. Coey [27]).

[Some two subnetwork amorphous magnetic structures.]

caractéristiques des verres de spin telles qu'elles apparaissent dans les alliages métalliques. Les verres de spin constituent une classe d'alliages dans lesquels des éléments magnétiques sont dilués dans une matrice cristalline non magnétique, en concentration suffisamment faible $(\simeq 1 \%)$ pour que leur répartition puisse être considérée comme totalement aléatoire. Ils se caractérisent par l'absence d'ordre magnétique à longue distance, même à très basse température ; seulement, en dessous d'une certaine température $T_{\mathrm{g}}$ caractéristique, à cause de leurs interactions mutuelles, les moments se bloquent dans des directions aléatoires de l'espace. Le gel des moments magnétiques en dessous de $T_{\mathrm{g}}$ conduit à des propriétés physiques très caractéristiques :

a) L'apparition d'un maximum très aigu de susceptibilité, mesurée en champ alternatif très faible (Fig. 12). Cette pointe ou cusp s'arrondit lorsque le champ de mesure croît.

$\beta)$ Pour $T>T_{\mathrm{g}}$, la susceptibilité suit une loi de Curie-Weiss; elle prend une valeur finie non nulle à $0 \mathrm{~K}$. La valeur de $\theta_{\mathrm{p}}$ est voisine de 0 .

$\gamma)$ La température de gel est proportionnelle à la concentration en impuretés.

$\delta$ ) En dessous de $T_{\mathrm{g}}$, apparaissent des phénomènes irréversibles, comme dans le cas des spéromagnétiques, avec des effets de thermorémanence, fonction

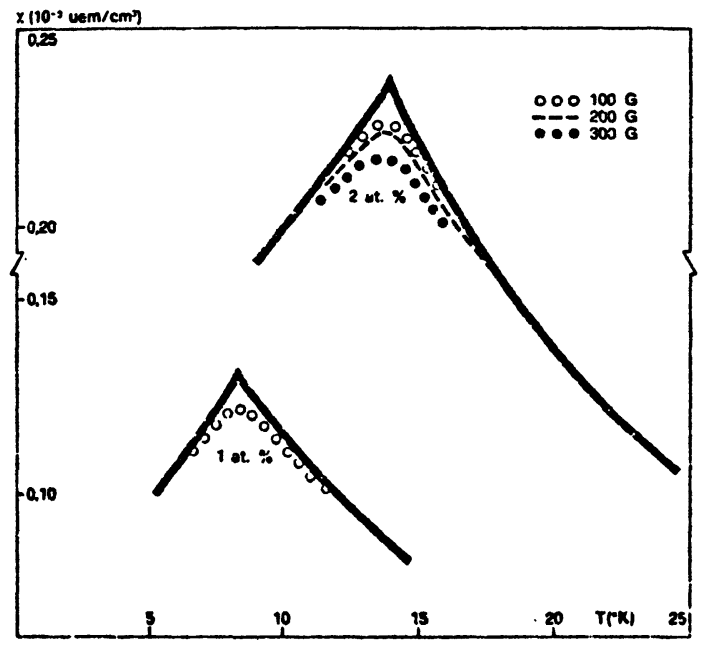

.Fig. 12. - Susceptibilité de AuFe $1 \%$ et $2 \%$ en champ alternatif faible (trait plein). Le pic s'arrondit sous l'action d'un champ continu extérieur (d'après [52 et 53]).

[Susceptibility of AuFe $1 \%$ and $2 \%$ in low alternative field [52-53].]

de l'histoire thermique et magnétique de l'échantillon, et de l'hystérésis.

ع) Les aimantations rémanentes, TRM et IRM définies comme précédemment dépendront du temps et sont toujours faibles et très anisotropes en champ faible. Elles se saturent sous des champs sensiblement égaux et faibles (quelques kG) (Fig. 13) et l'ATR présente un maximum arrondi avant d'atteindre sa valeur à saturation.

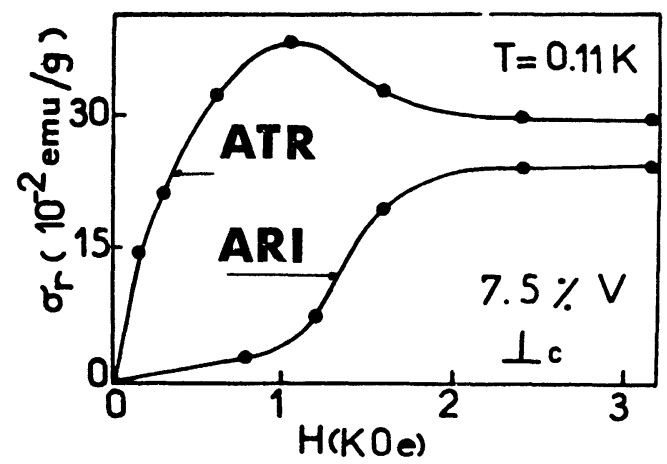

Fig. 13. - Aimantation thermorémanente et rémanente isotherme de $\left(\mathrm{Ti}_{1-x} \mathrm{~V}_{x}\right)_{2} \mathrm{O}_{3}$ (d'après Dumas [54]).

[TRM and IRM curves of $\left(\mathrm{Ti}_{1-x} \mathrm{~V}_{x}\right)_{2} \mathrm{O}_{3}$ [54].]

ל) Enfin, une propriété caractéristique des verres de spin, due à l'invariance du produit $x r^{3}$ ( $x$ étant la concentration en impuretés et $r$ la distance moyenne entre impuretés), est l'existence de lois d'échelle. Si l'on désigne par $M$ et $M R$ l'aimantation et l'aimantation rémanente, les quantités $\frac{M}{x}$ et $\frac{M R}{x}$ sont des fonctions universelles des variables réduites $\frac{H_{\mathrm{ext}}}{x}$ et $\frac{T}{x}$.

Ces lois d'échelle ne sont vérifiées que pour des concentrations faibles. Au-delà de quelques $\%$ en éléments magnétiques, elles ne sont plus respectées. Les écarts observés sont liés alors aux effets affectant 
le caractère aléatoire de la répartition des impuretés. On aborde alors le domaine du mictomagnétisme lié à certaines microinhomogénéités dans l'échantillon. Les propriétés physiques d'un mictomagnétique, toujours liées à l'histoire thermique et magnétique de l'échantillon différeront de celles des verres de spin :

$\alpha)$ par un maximum très large de la susceptibilité,

$\beta$ ) par des valeurs négatives de la température de Curie asymptotique relativement importantes,

$\gamma)$ par l'apparition de cycles d'hystérésis décalés sur l'axe des champs [55].

Au-dessous de la température de gel, apparaît de plus le phénomène de superparamagnétisme. Ces propriétés particulières tendent actuellement à être expliquées par l'apparition de clusters magnétiques interagissant entre eux; une large distribution de la taille des clusters et de leur temps de relaxation pourrait alors justifier le maximum très étalé de la susceptibilité. Cependant, comme le notent $\mathrm{H}$. Laville et
J. C. Bernier [55] à propos de leurs études sur les verres d'oxydes $\mathrm{BaO}-\mathrm{Fe}_{2} \mathrm{O}_{3}-\mathrm{Na}_{2} \mathrm{O}$ et $\mathrm{BaO}-\mathrm{Fe}_{2} \mathrm{O}_{3}$ $\mathrm{B}_{2} \mathrm{O}_{3}$, il semble qu'il n'existe pas de différence significative entre le mictomagnétisme observé dans les alliages cristallisés et dans celui des verres d'oxyde isolants. Dans ce dernier cas, l'interaction RKKY ne pouvant intervenir, il leur semble que les phénomènes observés pourraient être expliqués par la théorie des grains fins de Néel [56].

L'étude récente des interactions magnétiques dans les amorphes ioniques nécessite donc d'être approfondie en utilisant de nombreuses techniques concomitantes parmi lesquelles la RMN du fluor - susceptible de donner des indications sur la distribution des distances F-F dans les amorphes -, la diffusion des neutrons en dessus et en dessous de la température de gel afin de déterminer les fonctions de distribution radiale atomique et magnétique, et bien entendu la Spectrométrie Mössbauer occuperont une place privilégiée.

\section{Bibliographie}

[1] Rawson, H., Inorganic glass forming systems (Academic Press) 1967.

[2] Poulain, M., LuCas, J., Verres Refract. 32 (1978) 505.

[3] Stevic, S., Videau, J. J., Portier, J., Rev. Chim. Min. 15 (1978) 529.

[4] ZarzycKi, J., Phys. Chem. Glass. 12 (1971) 97.

[5] Shirk, B. T., Buessem, W. R., J. Am. Ceram. Soc. 53 (1970) 192.

[6] Fahmy, M., Park, M. J., Tumozawa, M., Mac Crone, R. K. Phys. Chem. Glass. 13 (1972) 21

[7] Moon, D. W., Aitken, J. M., Mac Crone, R. K., CielosZYK, G. S., Phys. Chem. Glass. 16 (1975) 91.

[8] Park, M. J., Mac Crone, R. K., J. Korean. Phys. Soc. 7 (1974) 73.

[9] Tanigawa, H., TanaKa, N., Osaka Kogyo Oyretsu Shikenoko Kiko 15 (1964) 285.

[10] Sarjeant, P. T., Roy, R., J. Am. Ceram. Soc. 50 (1967) 500

[11] Kantor, P., Revcolevski, A., Collongues, R., J. Mat. Sci. 8 (1973) 1359.

[12] Suzuki, T., Anthony, A. M., Mat. Res. Bull. 9 (1974) 745.

[13] Monteil, J. B., Bernier, J. C., Revcolevski, A., Mat. Res. Bull. 12 (1977) 235.

[14] Monteil, J. B., Padel, L., Bernier, J. C., J. Soliď State Chem. 25 (1978).

[15] Horie, O., Syono, Y., Makagawa, Y., Ito, A., Okamura, K., Yajima, S., Solid State Commun. 25 (1978) 423

[16] Chaumont, C., Boissier, J., Bernier, J. C., Rev. Int. Htes Temp. Refract. (1978) 15-23.

[17] Laville, H., Bernier, J. C., Sanchez, J. P., Solid State Commun. 27 (1978) 259

[18] Boyle, A. J. F., Kalvius, G. M., Gruen, D. M., Crifton, J. R., Macbeth, R. L., J. Physique Colloq. 32 (1971) C1-224.

[19] Lititerst, F. J., Kalvius, G. M., Boyle, A. J. F., J. Physique Colloq. 35 (1974) C6-403.

[20] Litterst, F. J., J. Physique Lett. 36 (1975) L-197.

[21] Ferey, G., Leclerc, A. M., de Pape, R., Mariot, J. P. VARRET, F., Solid State Commun. 29 (1979) 477.

[22] Ferey, G., Varret, F., Coey, J. M. D., J. Physics C 13 (1979) 531.

[23] Van Diepen, A. M., Popma, Th. J. A., J. Physique Colloq. 37 (1976) C6-755

[24] Popma, Th. J. A., van Diepen, A. M., Mat. Res. Bull. 9 (1974) 1119.

[25] Popma, Th. J. A., van Diepen, A. M., AIP Conf. Proc. 24 (1974) 123
[26] Fonteneau, G., Aliaga, N., Corre, O., Lucas, J., Rev. Chim. Min. 15 (1978) 537.

[27] Coey, J. M. D., J. Appl. Phys. 49 (1978) 1646.

[28] Kanamori, J., J. Phys. Chem. Solids 10 (1959) 87.

[29] Goodenough, J. B., Magnetism and the Chemical bond (Interscience Ed.) 1963

[30] Kobe, S., Ferchmin, A. R., J. Mater. Sci. 12 (1977) 1713.

[31] Toulouse, G., Comm. Phys. 2 (1977) 115.

[32] Vannimenus, J., Toulouse, G., J. Phys. C 10 (1977) L 2537.

[33] Kirkpatrick, S., Phys. Rev. B (1977).

[34] KURTZ, Dissertation Tubingen (1979).

[35] Sherrington, D., AIP Conf. Proc. 29 (1976) 224

[36] BINDER, K., Festkörperproblem 17 (1977) 55.

[37] Edwards, S. F., Anderson, P. W., J. Phys. F 5 (1975) 965.

[38] Sherrington, D., KirkPATrick, S., Phys. Rev. Lett. 35 (1975) 1792.

[39] Simpson, A. W., Wiss. Z. Tech. Univ. Dresden 23 (1974) 1027.

[40] Coey, J. M. D., Readman, P. W., Nature 246 (1973) 476 Earth. Planet Sci. Lett. 21 (1973) 45.

[41] Reboulllat, J. P., Lienard, A., Coey, J. M. D., ArreseBoggiano, R., Chappert, J., Physica 86-88B (1977) 773.

[42] Chappert, J., Arrese-Boggiano, R., Coey, J. M. D., J. Magnetism Magnetic Materials.

[43] JaCobs, I. S., BeAN, C. P., Magnetism III Rado-Shull Ed. 271 (1963) (Academic Press, N.Y.).

[44] Coey, J. M. D., Communication privée.

[45] Chaudhari, P., Cuomo, J. J., Gambino, R. J., IBM. J. Res. Dev. 17 (1973) 66

[46] Heiman, N., Lee, K., Phys. Lett. 55A (1975) 297.

[47] Forrester, D. W., Pala, W. P., Segnan, R., Amorphous Magnetism II (1977) 135 ;

Levy, R. A., Hasegawa, R., Ed. (Plenum 1977).

[48] Rhyne, J. J., Schnelling, J. H., Koon, N. C., Phys. Rev. B 10 (1974) 4672

[49] Coey, J. M. D., Chappert, J., Rebouillat, J. P., Wang, T. S., Phys. Rev. Lett. 36 (1976) 1061.

[50] Bhattacharjee, A. K., Julien, R., Zuckerman, M. J., J. Phys. F 7 (1977) 393.

[51] BeCK, P. A., Progress in Material Science 23 (1978) 1.

[52] Cannella, V., Mydosh, J. A., Phys. Rev. B 6 (1972) 4220.

[53] Rammal, R., La Recherche 8 (1977) 1093.

[54] Dumas, J., Thèse de Doctorat es Sciences Physiques Grenoble (1978).

[55] Laville, H., Bernier, J. C., Preprint.

[56] NeEl, L., Ann. Geophys. 5 (1949) 99. 